\title{
Evolution of Mound Morphology in Reversible Homoepitaxy on $\mathrm{Cu}(100)$
}

J.-K. Zuo

Missouri State University

J. F. Wendelken

Follow this and additional works at: https://bearworks.missouristate.edu/articles-cnas

\section{Recommended Citation}

Zuo, J-K., and J. F. Wendelken. "Evolution of mound morphology in reversible homoepitaxy on Cu (100)." Physical review letters 78, no. 14 (1997): 2791.

This article or document was made available through BearWorks, the institutional repository of Missouri State University. The work contained in it may be protected by copyright and require permission of the copyright holder for reuse or redistribution.

For more information, please contact BearWorks@library.missouristate.edu. 


\title{
Evolution of Mound Morphology in Reversible Homoepitaxy on Cu(100)
}

\author{
J.-K. Zuo ${ }^{1}$ and J.F. Wendelken ${ }^{2}$ \\ ${ }^{1}$ Department of Physics and Astronomy, Southwest Missouri State University, Springfield, Missouri 65804 \\ ${ }^{2}$ Solid State Division, Oak Ridge National Laboratory, Oak Ridge, Tennessee 37831
}

(Received 17 September 1996)

\begin{abstract}
Evolution of mound morphology in reversible homoepitaxy on $\mathrm{Cu}(100)$ was studied via spot-profileanalysis (SPA) LEED and scanning tunneling microscopy. The mound separation shows coarsening vs growth time with $L(t) \sim t^{1 / 4}$, in support of theory based on capillarity between mounds. The growth ultimately reaches a steady state characterized by a selected mound angle of $\sim 5.6^{\circ}$. We suggest that this results from a downhill current driven by step edge line tension in balance with an uphill current due to the Schwoebel barrier effect. Also, we have clarified the interpretation for the evolution of the SPA-LEED profile from a ring structure to a single time-invariant peak. [S0031-9007(97)02851-2]
\end{abstract}

PACS numbers: 68.55.Jk, 61.14.Hg, 61.16.Ch

Recently, a number of epitaxial growth experiments have shown a pyramidlike mound morphology with a welldefined mound separation and a selected mound slope [1-6]. The origin of this phenomenon is ascribed to a growth instability caused by the step barrier (or Schwoebel barrier) which resists step-down diffusion of deposited atoms [7]. As a result, the probability for the nucleation of upper-level islands onto lower-level islands is enhanced. Repeated application of this process in successive layers leads to the observed pyramidlike mound morphology. As deposition proceeds, the mounds grow bigger and steeper (i.e., unstable), and coalescence will occur with the filling of gaps between mounds and development of new top layers. This results in the coarsening of the island size distribution, i.e., the distribution becomes dominated by larger islands at the expense of smaller ones, as characterized by the increase in the average mound separation $L(t)$ with growth time $t$. In general, $L(t)$ is found to follow a power law [3-6,8-10],

$$
L(t) \propto t^{n},
$$

with the exponent $n$ depending on the coarsening mechanism.

Mullins first obtained Eq. (1) with $n=1 / 4$ by solving a continuity equation, $\partial h / \partial t=-\nabla^{4} h$, where $h$ is the surface height and the $\nabla^{4} h$ term describes capillary-induced mound coalescence which eliminates smaller mounds in favor of larger ones [10]. Recently, Siegert and Plischke [8] and Hunt et al. [9] also obtained $n \sim 0.25$ from numerical integration of the continuity equation with additional terms incorporating the Schwoebel barrier effect. This kind of coalescence only occurs when a local equilibrium can be established between diffusing atoms and growing mounds. The process requires detachment of an atom from the step edges of small mounds in a time scale roughly equal to $\sim 1 / F$ ( $F$ is the flux), i.e., a reversible growth. However, if such a detachment is not allowed, i.e., for an irreversible growth, Stroscio et al. obtained $n \sim 0.18$, for which they introduced a term for a so-called local "corner" free energy in the continuity equation in- stead of the $\nabla^{4} h$ term [4]. This exponent agrees with their measured value of $n \sim 0.16$ from the $\mathrm{Fe} / \mathrm{Fe}(100)$ growth at a temperature $T=293 \mathrm{~K} \mathrm{[4]}$ at which the growth has previously been shown to be irreversible [11], and also is supported by a recent kinetic Monte Carlo simulation by Amar and Family [12]. The other recent experiments on $\mathrm{Fe} / \mathrm{Mg}(100)$ [5], Ge/Ge(100) [3], and $\mathrm{Rh} /$ mica [6] show $n \sim 0.23,0.4$, and 0.33 , respectively. These values of $n$ are inconsistent with each other and also with any existing models except for $n \sim 0.23$. However, these systems are either anisotropic or heteroepitaxial, thus introducing extra complexities which are not considered in existing theories. Also, in these experiments it is not clear whether the growth is reversible or irreversible. From Monte Carlo simulations on a simple square lattice system [13], it is indeed found that the value of $n$ is sensitive to the growth temperature $T$, but not to the step barrier in a range of $0.05-2.0 \mathrm{eV}$. Another feature in the mound growth is the selected mound slope. It is argued [4,8] that this results from a balance between an uphill current formed by reflection of diffusing atoms by the Schwoebel barrier at a descending step edge and a downhill current described by a phenomenological term $\propto m^{3}$, where $m$ is the mound slope. The microscopic origin of this $m^{3}$ term is unclear at present. Current understanding of mound growth is still very limited, particularly for the case of reversible growth.

In this Letter, we report our study of unstable mound growth on $\mathrm{Cu} / \mathrm{Cu}(100)$ in the reversible growth regime near room temperature, using spot-profile-analysis lowenergy electron diffraction (SPA-LEED) and scanning tunneling microscopy (STM). The study includes the coarsening mechanism and the microscopic origin of the downhill current responsible for the selected mound slope in reversible growth. The other purpose of this study is to interpret a single time-invariant SPA-LEED profile at large thicknesses and to distinguish this from a self-affine scenario.

SPA-LEED and STM experiments were performed in different ultrahigh vacuum chambers with similar base pressures $\left(\sim 4.0 \times 10^{-11}\right.$ Torr $)$. The $\mathrm{Cu}(100)$ substrate 
was cleaned by $\mathrm{Ne}^{+}$sputtering and then annealed at $\sim 500{ }^{\circ} \mathrm{C}$ for $5-10 \mathrm{~min}$. After the cleaning, no impurities were detected in situ with Auger electron spectroscopy. The average terrace size on the clean $\mathrm{Cu}(100)$ surface is determined by STM to be $\sim 850 \AA$. However, STM imaging is usually performed on terraces larger than $2000 \AA$. Copper was evaporated from a $99.9999 \%$ pure $\mathrm{Cu}$ source onto the substrate at $T \sim 303 \mathrm{~K}$ for the SPA-LEED experiment and at $\sim 299 \mathrm{~K}$ for the STM experiment, with the same deposition rate of $F \sim 0.0208$ monolayer (ML)/s. Temperature was measured with the thermocouple attached on or near the sample for the SPALEED and STM studies, respectively.

$\mathrm{Cu} / \mathrm{Cu}(100)$ provides a simple square lattice system, ideal for testing existing models without extra complexity, and its many aspects have been investigated [2,14-17]. For example, we have observed with STM that small islands in the top layer of typical size $\sim 40 \AA$ gradually disappear over a period of $\sim 75 \mathrm{~min}$ at room temperature. From this it can be calculated that the average time for an atom detachment is $\sim 18 \mathrm{sec}$ which is much less than $1 / F \sim 48 \mathrm{sec}$. This clearly indicates that the $\mathrm{Cu} / \mathrm{Cu}(100)$ growth near room temperature is reversible, a necessary condition for the capillary-induced coalescence of mounds. Also, the Schwoebel barrier for the system does exist as evidenced by faceting at low temperatures [2].

LEED spots at antiphase conditions during the $\mathrm{Cu} /$ $\mathrm{Cu}(100)$ growth start with a ring structure plus a sharp central peak. The diffraction ring is known to result from a well-defined island separation $L$ due to a limited adatom diffusion length [14,15]. Shown in Fig. 1 is a set of the (00) angular profiles, scanned across the diffraction ring along the [110] direction, for deposition at different thicknesses and $T \sim 303 \mathrm{~K}$. The central peak oscillates and damps out over several layers, indicating a multilayer island (or mound) growth. In the meantime, the diameter of the diffraction ring $L^{*}(\propto 1 / L)$, which is given by the separation of the two side peaks in Fig. 1, decreases with deposition until a thickness of $\sim 20 \mathrm{ML}$, implying a coarsening of mounds (see STM images in Fig. 3 below). The coarsening starts after the island coalescence has occurred in the submonolayer regime. Figure 2 is a $\log -\log$ plot of $L^{*}$ vs growth time $t$ (or thickness $=F t$ ) in a range of 1.1-20 ML. The solid line is a leastsquares fit of Eq. (1) to the data which gives $n=0.25 \pm$ 0.01 , in excellent agreement with the capillary-induced coalescence mechanism [8-10].

Another interesting phenomenon shown in Fig. 1 is that when the thickness increases above $\sim 20 \mathrm{ML}$ the split peaks gradually broaden and merge into a single broad peak. This single peak broadens further with deposition until reaching a steady shape above $\sim 100 \mathrm{ML}$. This steady profile is similar to that observed for the $\mathrm{Fe} / \mathrm{Fe}(100)$ growth with SPA-LEED, in which a self-affine growth was concluded [18]. The questions raised now are: What is the real surface morphology at large thicknesses? Why does the diffraction profile evolve into a

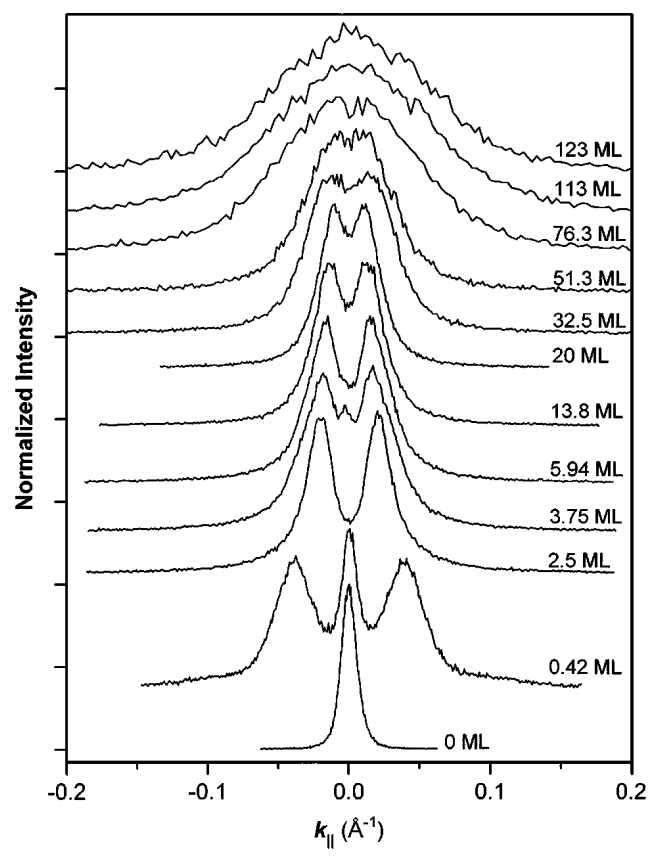

FIG. 1. SPA-LEED angular profiles measured for deposition at different thicknesses (ML) and $T \sim 303 \mathrm{~K}$, where $k_{\|}$is the scattering vector parallel to the surface.

single broad peak instead of the ring structure if the moundlike morphology persists at large thicknesses? These questions can be answered by examining STM data. Figure 3 is a set of STM images at different thicknesses and $T \sim 299 \mathrm{~K}$. From the images we can see the evolution of the mound morphology and the coarsening of the mound size distribution. One important point should be noted: As the upper-level islands develop, the lower-level islands become interconnected. This has the fact that, while there is a well-defined mound separation for the upper layers, this well-defined separation is lost for the lower layers. SPA-LEED is at a nearly normal incidence, and incident electrons can reach and be scattered from all exposed surface atoms even in deep valleys. The shape of the angular profile will depend on the relative contributions of scattering from upper and

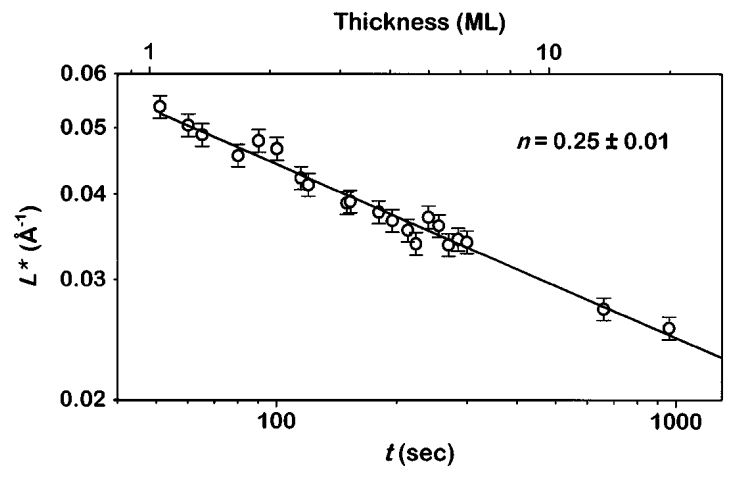

FIG. 2. A log-log plot of the diameter of the diffraction ring $L^{*}(t)$ vs growth time $t$ or thickness $(=F t)$. The solid line is a least-squares fit of Eq. (1) to the data, and the slope gives the coarsening exponent $n$. 


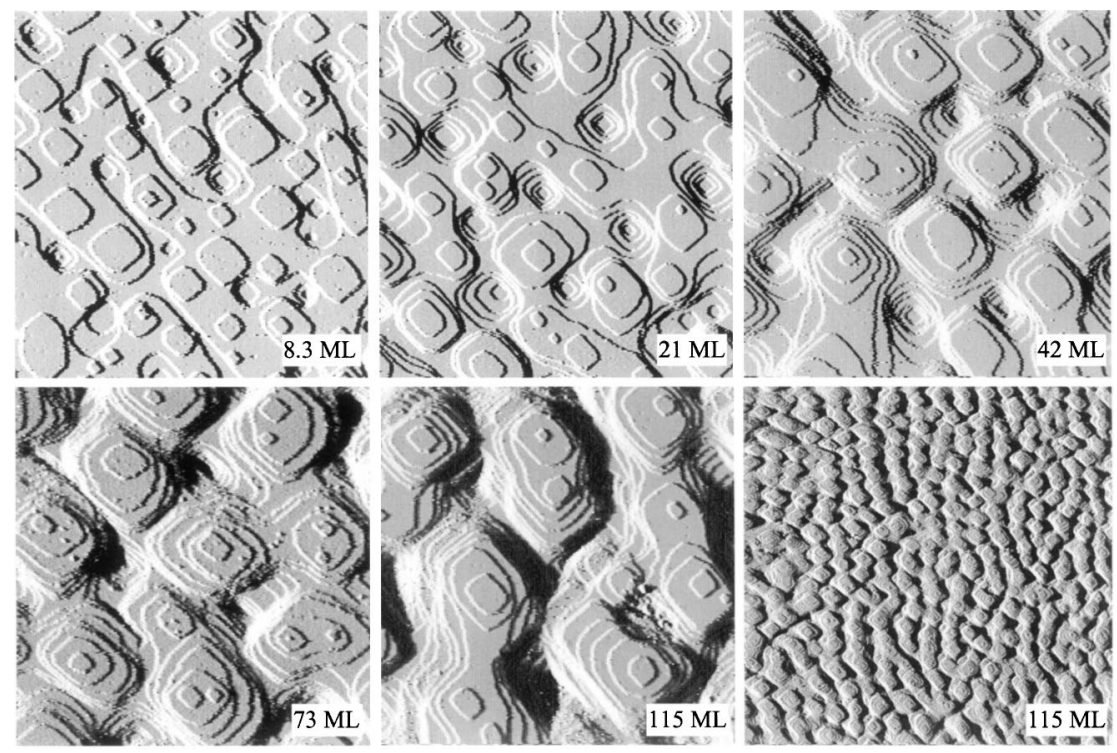

FIG. 3. STM images of the first derivative measured for deposition at different thicknesses and $T \sim 299 \mathrm{~K}$. The first five images show an area $1000 \times 1000 \AA^{2}$ while the last one is $5000 \times 5000 \AA^{2}$. Small clusters in some of the images may be attributed to mobile small $\mathrm{Cu}$ clusters. Some specks are noise enhanced by the derivative mode.

lower layers. Since the upper layers necessarily constitute a smaller fraction of the total terrace area than the lower layers after $\sim 20 \mathrm{ML}$, the SPA-LEED profile in this case mainly reflects the distribution of irregular terrace sizes in the lower layers with a corresponding change to a single broad peak. The steady profile above $100 \mathrm{ML}$ indicates that the step density becomes time invariant; in other words, the mound slope stabilizes, rather than developing a self-affine morphology. By estimating the average terrace size $(\ell \sim 43 \AA)$ from the full width at half maximum (FWHM) of the profile in the steady growth regime, the saturated average mound angle is calculated to be $\phi_{0}=\tan ^{-1}(d / \ell) \sim 2.4^{\circ}$, where $d=1.8077 \AA$ is the atomic step height.

In contrast to LEED, RHEED (reflection high-energy electron diffraction) is at a glancing incidence, and incident electrons can only hit and be scattered from the top few atomic layers. Because of the existence of a well-defined mound separation for the upper layers, the RHEED profile is expected to remain split at large thicknesses. This has actually been demonstrated in a simulation, by Amar and Family [19], for the SPA-LEED and RHEED profiles from the irreversible $\mathrm{Fe} / \mathrm{Fe}(100)$ mound growth with a deposition rate of $\sim 0.0257 \mathrm{ML} / \mathrm{s}$ (comparable to ours). They found that the SPA-LEED profile turns into a single steady peak after $\sim 20 \mathrm{ML}$ but the RHEED profile remained split. They interpret the loss of the ring structure in the SPA-LEED profile at large thicknesses to be the result of mound edge roughening (kinks) due to limited edge diffusion, and, therefore, not necessarily due to the loss of a characteristic length among mounds. In our reversible growth, the edge diffusion is sufficient, as implied by the smooth step edges in STM images, thus edge roughening is not relevant here.
Now, we will quantify the time dependence of the average mound separation $L(t)$ and mound slope $m(t)$ in a much wider thickness range of $0.42-145 \mathrm{ML}$ from STM images. To do so, we calculated a height-height correlation function from STM images, which is defined as $G(r)=\langle\tilde{h}(r) \tilde{h}(0)\rangle$, where $\tilde{h}(r)=h(r)-\langle h\rangle$ is the height relative to the mean height $\langle h\rangle, r$ is the lateral distance, and the $\langle\ldots\rangle$ is the spatial average over the surface. The average mound separation and slope are determined via $L=2 r_{c}$ and $m=w / r_{c}$ [4], where $r_{c}$ is the position of the first zero crossing of $G(r)$ and $w=[G(0)]^{1 / 2}$ is the rms surface roughness. Using this method to determine $L$ gives a much better statistical average than the method of counting the number density of mounds $\left(L=N^{-1 / 2}\right)$. The results are plotted in Fig. 4. The least-squares fit to the data gives the coarsening exponent $n=0.23 \pm$ 0.01 , consistent with the SPA-LEED measurement and supporting the capillary-induced coalescence mechanism. Also consistent with the SPA-LEED measurement, the average mound slope $m$ in Fig. 4 increases gradually with growth time and saturates at a value of $m_{0}=0.042$ (or $\phi_{0}=2.4^{\circ}$ ) after $\sim 90 \mathrm{ML}$. In contrast, saturation of the mound slope occurs much more rapidly (at $\sim 3 \mathrm{ML}$ ) for irreversible growth of $\mathrm{Fe} / \mathrm{Fe}(100)$ [4]. However, the STM images at large thicknesses in Fig. 3 suggest an underestimate of the mound slope obtained. This is because the STM images show some mounds with flat tops containing one or two small islands. Thus, the mound slope obtained above is an average value, rather than just for the mound step walls. Although similar mound formation also exists in irreversible growth of $\mathrm{Fe} / \mathrm{Fe}(100)$ [4] and in kinetic Monte Carlo simulations [19], the population of flat tops here is higher. This is because in reversible growth islands which nucleate on the top terrace can also undergo dissociation processes. 


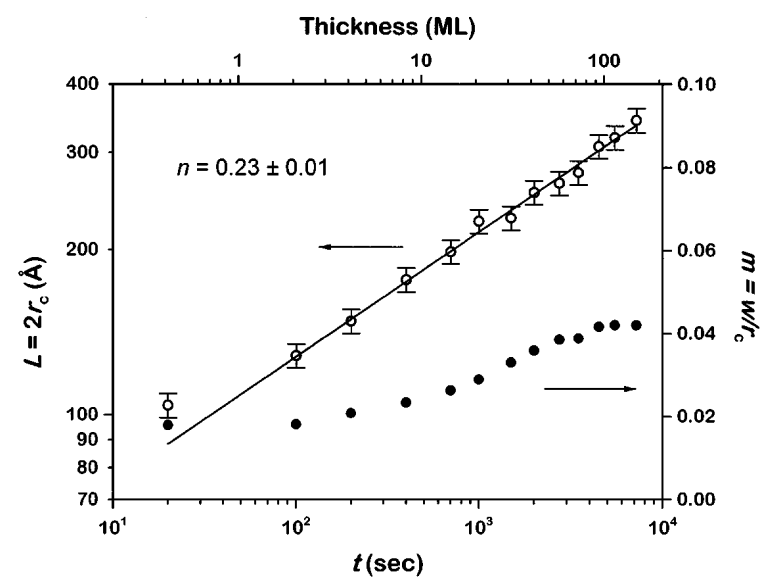

FIG. 4. A plot of the average mound separation $L$ (left axis) and average mound slope $m$ (right axis) vs growth time $t$ or thickness. The solid line is a least-squares fit of Eq. (1) to the data which gives $n$.

Thus, the probability to form a stable island on the top terrace will be reduced in our case as compared to the irreversible growth case. Dissociation of top terraces during the time (10-15 $\mathrm{min}$ ) between deposition and STM imaging is negligible as indicated by a time sequence of STM images. To eliminate this effect, we have measured the mound step wall angles directly from STM images and found that the average step wall angle is saturated after $\sim 90 \mathrm{ML}$ with a saturation angle of $\sim 5.6 \pm 1.3^{\circ}$. It will be more reasonable to use this angle as the selected mound angle here. In comparison, for an irreversible growth of $\mathrm{Cu} / \mathrm{Cu}(100)$ at low temperatures [2], the mound sides were found to consist of the (113) facet at $160 \mathrm{~K}$ and the (115) facet at $200 \mathrm{~K}$. Apparently, these mound angles are much larger than observed here, indicating the existence of a smoothing mechanism in our reversible growth.

At this point, one more question needs to be answered: What process competes with the uphill current associated with the Schwoebel barrier to limit the mound slope to a much smaller value than that observed for irreversible growth $[2,4]$ ? To see this, we have measured a time sequence of STM images after stopping the deposition at $140 \mathrm{ML}$. We found that the average terrace width expands at a rate of $\sim 0.2 \AA / \mathrm{min}$, but the step wall angle decreases more slowly at a rate of $\sim 0.01^{\circ} / \mathrm{min}$. This smoothing effect on the morphology during the 10-15 min between deposition and STM imaging is negligible. The physical origin of this smoothing mechanism was found by us on the same system [16] to be driven by the line tension of curved step edges, for which the step free energy can be written as $\mu=\mu_{0}+\sigma / R$ [20], where $\mu_{0}$ is the straight step free energy and $\sigma$ is the edge line tension for a terrace of radius $R$. Therefore, in order to lower the free energy, atoms will be driven to migrate from up-terrace edges to downterrace edges in a mound, forming a net downhill current, $\quad J_{-}=\left(1-P_{r}\right) k \partial \mu / \partial R=-\left(1-P_{r}\right) k \sigma / R^{2} \sim$ $-\left(1-P_{r}\right) k \sigma / \ell^{2}=-\left(1-P_{r}\right) k \sigma m^{2}$, where $P_{r}$ is the probability per unit time that an atom which diffuses to the down step will be reflected, $k$ is a kinetic coefficient, and $\ell$ is the terrace width. Since $m \ll 1$ here, this term $\left(\propto \mathrm{m}^{2}\right)$ will be dominant over the phenomenological term $\left(\propto \mathrm{m}^{3}\right)$ mentioned above if it exists. The net uphill current in the steady growth regime can be written as $J_{+}=P_{r} F / 2 m$. From $J_{-}+J_{+}=0$, we obtain the selected mound slope, $m_{0}=\left[P_{r} F / 2\left(1-P_{r}\right) k \sigma\right]^{1 / 3}$. The smoothing kinetics described above could be faster during deposition since the newly deposited adatoms will be driven to migrate to descending terrace edges by a lateral pressure gradient caused by the line tension differences. After deposition, an atom first has to dissociate from the up-terrace edge before it can be driven to migrate to the down-terrace edge.

Finally, we discuss the kinetic roughening properties of this system. From STM images we calculated another height-height correlation function defined as $G^{\prime}(r)=$ $\left\langle[h(r)-h(0)]^{2}\right\rangle$ for which $G^{\prime}(r) \sim r^{2 \alpha}$ for $r \ll L[19]$. For mound growth, one expects $\alpha=1$ in the steady growth regime since there is only one length scale among mounds [8]. Also, the rms surface roughness, $w$, calculated above increases with growth time, as $w \sim t^{\beta}$. From a plot of $w$ and $\alpha$ vs $t$, we obtain $\beta \sim 0.45 \pm 0.01$, and $\alpha \sim 0.92$ in the steady growth regime. The value of $\alpha$ agrees with prediction, but the large $\beta$ is due to the slow saturation of the mound slope for reversible growth.

The work by J.K.Z. was supported by NSF Grant No. DMR-9311586, and the work by J.F.W. was supported by the Division of Materials Sciences, U.S. Department of Energy under Contract No. DEAC05-96OR22464 with Lockheed Martin Energy Research Corp.

[1] M. D. Johnson et al., Phys. Rev. Lett. 72, 116 (1994).

[2] H.-J. Ernst et al., Phys. Rev. Lett. 72, 112 (1994).

[3] J. E. Nostrand et al., Phys. Rev. Lett. 74, 1127 (1995).

[4] J. A. Stroscio et al., Phys. Rev. Lett. 75, 4246 (1995).

[5] K. Thürmer et al., Phys. Rev. Lett. 75, 1767 (1995).

[6] F. Tsui et al., Phys. Rev. Lett. 76, 3164 (1996).

[7] J. Villain, J. Phys. I (France) 1, 19 (1991); I. Elkinani et al., J. Phys. I (France) 4, 949 (1994).

[8] M. Siegert et al., Phys. Rev. Lett. 73, 1517 (1994).

[9] A. W. Hunt et al., Europhys. Lett. 27, 611 (1994).

[10] W. W. Mullins, J. Appl. Phys. 28, 333 (1957).

[11] J. A. Stroscio et al., Phys. Rev. B 49, 8522 (1994).

[12] J. G. Amar et al., Phys. Rev. B 54, 14742 (1996).

[13] P. Šmilauer et al., Phys. Rev. B 52, 14263 (1995).

[14] J.-K. Zuo et al., Phys. Rev. Lett. 72, 3064 (1994).

[15] H. Dürr et al., Surf. Sci. 328, L527 (1995).

[16] J.-K. Zuo et al., Phys. Rev. Lett. 70, 1662 (1993).

[17] M. C. Bartelt et al., Langmuir 12, 217 (1996); Phys. Rev. Lett. 75, 4250 (1995).

[18] Y.-L. He et al., Phys. Rev. Lett. 69, 3770 (1992).

[19] J. G. Amar et al., Surf. Sci. 365, 177 (1996).

[20] F. Lançon et al., Kinetics of Ordering and Growth at Surfaces, edited by M. G. Lagally (Plenum Press, New York, 1990), p. 369. 